Proceedings of the 5th Symposium on Physics in Economics and Social Sciences, Warszawa, Poland, November 25-27, 2010

\title{
Model of Communities Isolation at Hierarchical Modular Networks
}

\author{
P. Kondratiuk And J.A. HoŁyst
}

Faculty of Physics, Center of Excellence for Complex Systems Research, Warsaw University of Technology, Koszykowa 75, PL-00-662 Warsaw, Poland

The model of community isolation was extended to the case when individuals are randomly placed at the nodes of hierarchical modular networks. It was shown that the average number of blocked nodes (individuals) increases in time as a power function, with the exponent depending on the network parameters. The distribution of the time when the first isolated cluster appears is unimodal, non-gaussian. The developed analytical approach is in a good agreement with the simulation data.

PACS: 89.75.Hc, 02.50.--r, 89.75.-k, 89.75.Da, 89.75.Fb

\section{Introduction}

Recently, hierarchical systems have been attracting attention of scientists working in the field of complex networks [1-5]. In fact, many real networks are hierarchically organized, e.g. the World Wide Web network, the actor cooperation network, or the semantic web [1]. Dynamics at such networks can be qualitatively and quantitatively different from that at regular lattices (see [2-4]).

The Ising model at networks of hierarchical topology was studied by Komosa and Hołyst [2]. The analyzed parameters were, among others, the magnetization, the magnetic susceptibility, the critical temperature, and the correlations of magnetization between different hierarchies. It was shown that the critical temperature is a power function of the network size, as well as of the ratio $\frac{\left\langle k^{2}\right\rangle}{\langle k\rangle}$, where $k$ stands for a node degree.

Opinion formation in hierarchical organizations was studied by Laguna et al. [3]. Agents, belonging to various authority strata, try to influence each other's opinions. The probability that an opinion of an agent of a certain authority prevails in the community, depends on the size distribution of the authority strata. Phase diagrams can be obtained, where each phase corresponds to a distinct dominant stratum (or a sequence of the strata, with the decreasing probability of prevailing).

Fashion phenomena at hierarchical networks were studied by Galam and Vignes [4]. Interactions were imposed between social groups at different levels of hierarchy. The renormalization group approach was used to find the optimal investment level of the producer and to assess the influence of counterfeits on the probability of the new product success.

One of the fundamental topics in social dynamics are conflict situations and many different sociophysical approaches [6-8] or prisoner's dilemma-type games [9] have been proposed. Most such models concentrate on the problem of influence of neighbors on an individual agent's internal variable [10]. Recently a qualitatively different model has been introduced by Sienkiewicz and Hołyst [11]. The key idea behind that model is that isola- tion of communities makes them suffer and even extinct - a phenomenon often observed in ecology [12] and sociology [13-14].

The model of communities isolation has already been studied for chains, hypercubic, random and scale-free networks $[11,15]$. In this paper we extend it to the hierarchical networks proposed at [1].

\section{Hierarchical networks}

The model of hierarchical networks was proposed by Ravasz and Barabási [1] and extended by Suchecki and Hołyst [5]. Such networks are characterized by three parameters, determining their structure:

- The degree of hierarchy $h \in \mathbb{N} \cup\{0\}$

- The distribution $P_{M}(m)$, where $m \in \mathbb{N}$, determining the number of nodes at each level of hierarchy (in particular, the size of the cliques at the lowest level of hierarchy is equal to $m+1$ )

- The parameter determining the density of edges $p \in$ $[0,1]$

Two models (referred to as the P1 and PD models) were analyzed, which differ in the density of edges. Each network has a central node, referred to as the center of hierarchy. A network of hierarchy $h=0$ is a complete graph of size $m+1$ ( $m$ is a random number, chosen with probability $\left.P_{M}(m)\right)$. Here, the center of hierarchy, due to the symmetry, can be an arbitrary node. In order to construct a network of hierarchy $h>0$, one has to construct $m+1$ subnetworks of hierarchy $h-1$ and choose one of them as central. Its center of hierarchy, node $v$, becomes the center of hierarchy of the whole network. Afterwards, new connections (edges) are created: for each node $w$ of the $m$ remaining subnetworks a connection (edge) $(v, w)$ is created with probability $p$ (in the case of the $\mathrm{P} 1$ model) or $p^{h}$ (in the case of the PD model). Sample networks created this way are shown in Fig. 1. Let us stress that the subnetworks do not have to be connected, especially if $p$ is small. 


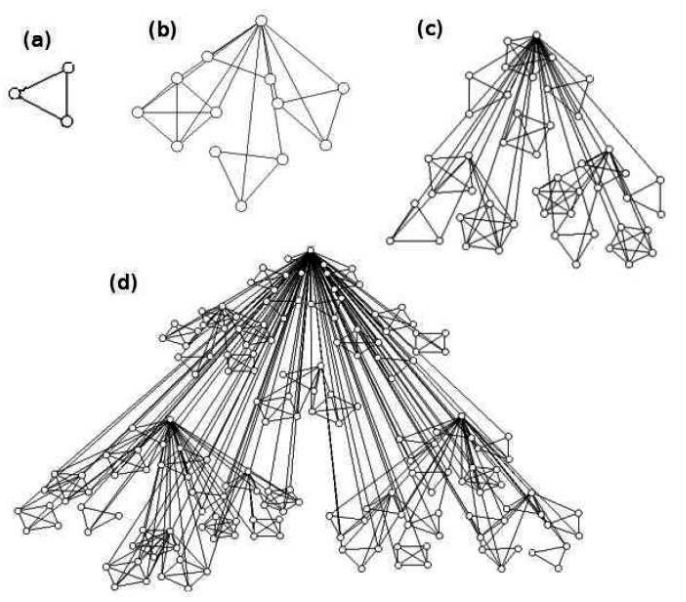

Fig. 1. Sample P1 networks with parameters $P_{M}(m)=\operatorname{Unif}(2,4), p=0.5$, and with different degrees of hierarchy: (a) $h=0$, (b) $h=1$, (c) $h=2$, (d) $h=3$.

Some basic properties of such networks can be concluded from the construction algorithm:

- For $h \in\{0,1\}$, as well as for $p \in\{0,1\}$, the P1 and PD models are equivalent.

- For $p=0$, the network consists of isolated cliques of size $m+1$ (where $m$ is a random variable).

- For $P_{M}\left(m^{\prime}\right)=\delta_{m^{\prime}, m}$ the number of nodes (vertices) of the network equals $N=(m+1)^{h+1}$.

Periodic oscillations in the degree distribution of such networks can be observed in the log-log scale. The period, the amplitude and the shape of the peaks depend on the network parameters [5].

In this paper only the case of $P_{M}\left(m^{\prime}\right)=\delta_{m^{\prime}, m}(m=$ const) was considered, which corresponds to the original Ravasz and Barabási model [1].

\section{Basic isolation model}

The model of communities isolation was proposed by Sienkiewicz and Hołyst [11]. The rules are similar to those of the game of Go. A number of communities compete with each other, settling nodes of a network. In each step a random empty node is chosen. It is then settled by a member of a randomly chosen community. A cluster of nodes occupied by one community becomes blocked when it gets surrounded by another community. The surrounded nodes are no more active in the game, i.e. they can not take part in surrounding other communities.

The case of communities competing at a chain was analyzed in [11]. Two functions describing the evolution were studied: the average number of blocked nodes over time and the mean critical time, i.e. the moment, when the first blocked cluster appears. In [15] the influence

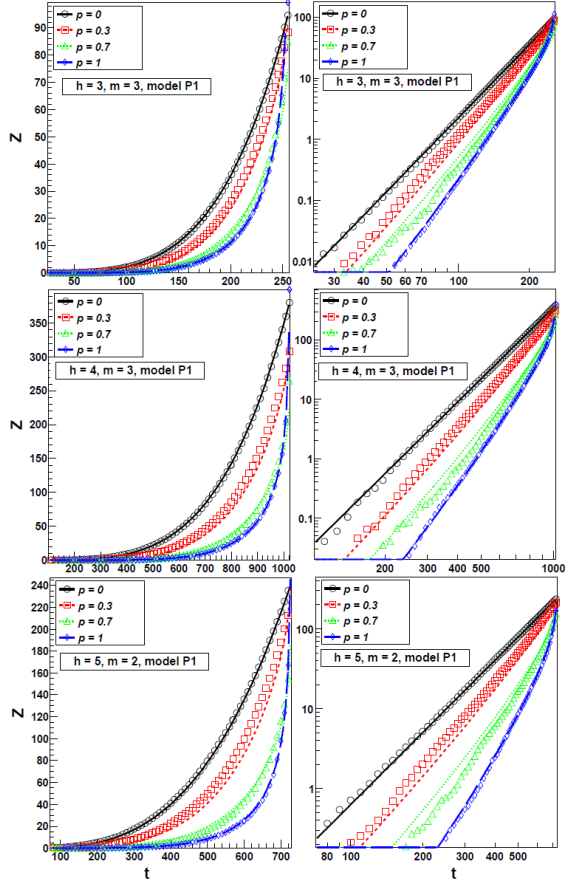

Fig. 2. The average number of blocked nodes, $Z(t)$, for various networks of the P1 model. Symbols correspond to the simulated data. Lines - analytical approximations (Eqs. (4.4), (4.7), and (4.9)). Left side - linear scale, right side - log-log scale.

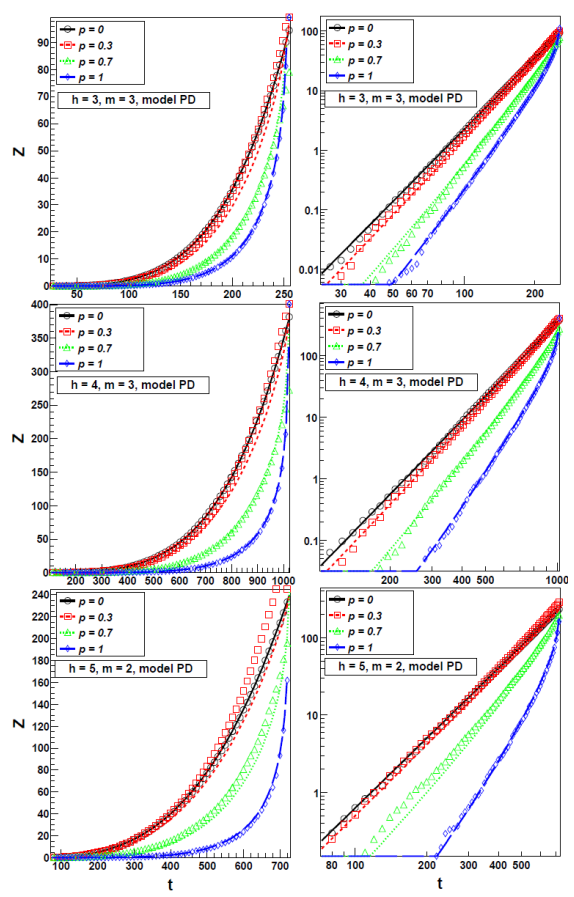

Fig. 3. The average number of blocked nodes, $Z(t)$, for various networks of the PD model. Symbols correspond to the simulated data. Lines - analytical approximations (Eqs. (4.4), (4.7), and (4.9)). Left side - linear scale, right side - log-log scale. 
of external bias was considered, i.e. the settling rates of competing communities are different.

In this paper the case of two competing communities at P1 and PD hierarchical networks is considered. Two parameters are analyzed: the average number of blocked nodes $Z(t)$ and the critical time distribution $\operatorname{Pr}\left(t_{c}\right)$.

\section{Number of blocked nodes over time}

\subsection{Case $p=0$}

For $p=0$, the $\mathrm{P} 1$ and $\mathrm{PD}$ models are equivalent. The network consists of $N /(m+1)$ isolated cliques of size $m+1$. In such case the average number of blocked nodes $Z(t)$ evolves the following way:

$$
\left\{\begin{array}{l}
Z(0)=0 \\
Z(t+1)=Z(t)+\sum_{i=1}^{m} i p_{i},
\end{array}\right.
$$

where $p_{i}$ denotes the probability that in the $(t+1)$ th step $i$ nodes will be blocked,

$$
p_{i}=\left(\frac{t}{2 N}\right)^{m}\left(\begin{array}{c}
m \\
i
\end{array}\right) \text {. }
$$

After short algebra we obtain

$$
\left\{\begin{array}{l}
Z(0)=0 \\
Z(t+1)=Z(t)+\frac{m}{2}\left(\frac{t}{N}\right)^{m} .
\end{array}\right.
$$

The solution of this recursive equation is a $(m+1)$ th degree polynomial, which can be approximated by substituting the sum with the integral:

$$
\begin{aligned}
& Z(t)=\sum_{i=0}^{t-1} \frac{m}{2}\left(\frac{i}{N}\right)^{m} \approx \int_{0}^{t} \frac{m}{2}\left(\frac{x}{N}\right)^{m} \mathrm{~d} x \\
& =\frac{m}{m+1} \frac{t^{m+1}}{2 N^{m}} .
\end{aligned}
$$

As one can see, $Z(t)$ is a power function. The exponent $\beta$ depends only on the $m$ parameter, $\beta=m+1$.

$$
\text { 4.2. Case } p=1
$$

In this case the P1 and PD models are also equivalent. For the networks of hierarchy $h=1$ :

$$
\begin{aligned}
& Z^{(1)}(t)=\rho_{0}\left(\rho_{1}^{m} \frac{m}{2}+m \rho_{1}^{m+1} \frac{m+1}{2}\right) \\
& =\frac{1}{2} m \rho_{0} \rho_{1}^{m}\left(1+(m+1) \rho_{1}\right),
\end{aligned}
$$

where $\rho_{i}$ denotes the reduced density:

$$
\rho_{i}=\rho_{i}(t) \equiv \begin{cases}0 & \text { for } t<i \\ \frac{t-i}{N-i} & \text { for } t \geq i\end{cases}
$$

For the networks of higher hierarchies, $h \geq 1$, a recursive equation approximating $Z^{(h)}(t)$ can be derived. The idea behind the formulas is as follows: a clique can only be blocked if all the nodes of higher hierarchies neighboring with it are filled. Therefore $Z^{(h)}(t)=0$ if the center of hierarchy of the network (which neighbors with all the other nodes) is empty. In the opposite case, $Z^{(h)}(t)$ depends on $Z^{(h-1)}(t)$, which describes each of the $m+1$ subnetworks.

$$
\left\{\begin{aligned}
Z_{i}^{(1)}(t) & =\frac{1}{2} m \rho_{i} \rho_{i+1}^{m}\left(1+(m+1) \rho_{i+1}\right) \\
Z_{i}^{(h)}(t) & =Z_{i}^{(h-1)}(t)+\frac{1}{2} m \rho_{i} Z_{i+1}^{(h-1)}(t) \\
& +\frac{1}{4} \rho_{i} \rho_{i+1} \rho_{i+2}^{(m+1)^{h}-1}\left((m+1)^{h}+1\right) \\
Z^{(h)}(t) & \equiv Z_{0}^{(h)}(t)
\end{aligned}\right.
$$

This equation can only be solved numerically. The solutions are presented in Figs. 2 and 3. It can be noticed that within a long period of time $t, Z^{(h)}$ can be approximated by a power function

$$
Z^{(h)}(t) \propto t^{\beta}
$$

with reasonable accuracy. The $\beta$ exponents are higher than in the case of $p=0$. In fact, they are close to $m+4$.

\subsection{General case}

An analytical approximation of $Z^{(h)}(t)$ for networks with higher hierarchies $(h>1)$, if the parameter $p$ is different from zero and one, is far more difficult. Instead of searching for such a formula, an alternative approach was chosen. It was assumed that $Z^{(h)}(t)$ can be estimated from the proportion

$$
\frac{\log Z_{p=0}^{(h)}(t)-\log Z^{(h)}(t)}{\log Z^{(h)}(t)-\log Z_{p=1}^{(h)}(t)} \approx \frac{f(p, h)}{1-f(p, h)},
$$

where $f(p, h) \in[0,1]$ should be an increasing function of $p$ which, while not being too complicated, would give a reasonable approximation for the widest possible ranges of $p$ and $h$. It turned out that in the case of the P1 model, choosing $f(p, h)=p$ results in a good agreement of the $Z^{(h)}(t)$ function with the simulation data. For the PD model, $f(p, h)=p^{\frac{h}{2}}$ is a good choice.

\section{Critical time distribution}

\subsection{Case $p=0$}

As it was previously mentioned, in the case of $p=0$, the network consists of $N /(m+1)=(m+1)^{h}$ isolated cliques of $m+1$ nodes. In order to find the distribution of the critical time (i.e., the moment when the first blocked cluster appears), one has to consider the probability that at time $t$ there are no blocked nodes yet. It means that at time $t$ the only completely filled cliques are those filled with members of one community, which leads to the formula

$$
\operatorname{Pr}\left(t_{c}>t\right)=\left(1-\alpha\left(\frac{t}{N}\right)^{m+1}\right)^{\frac{N}{m+1}},
$$

where $\alpha \equiv 1-2^{-m}$. The cumulative critical time distribution can be immediately obtained as

$$
\operatorname{Pr}\left(t_{c} \leq t\right)=1-\left(1-\alpha\left(\frac{t}{N}\right)^{m+1}\right)^{\frac{N}{m+1}},
$$

as well as the critical time distribution in the approximation of continuous time:

$$
\operatorname{Pr}\left(t_{c}=t\right)=\operatorname{Pr}\left(t_{c} \leq t\right)-\operatorname{Pr}\left(t_{c} \leq t-1\right)
$$




$$
\begin{aligned}
& \approx \frac{\mathrm{d}}{\mathrm{d} t} \operatorname{Pr}\left(t_{c} \leq t\right) \\
& =\alpha\left(1-\alpha\left(\frac{t}{N}\right)^{m+1}\right)^{\frac{N}{m+1}-1}\left(\frac{t}{N}\right)^{m}
\end{aligned}
$$

The mean critical time can be also calculated analytically:

$$
\begin{aligned}
\left\langle t_{c}\right\rangle & =\int_{0}^{N} t \operatorname{Pr}\left(t_{c}=t\right) \mathrm{d} t \approx \int_{0}^{N} t \frac{d}{\mathrm{~d} t} \operatorname{Pr}\left(t_{c} \leq t\right) \mathrm{d} t \\
& =\frac{N}{m+1} \alpha^{-\frac{1}{m+1}}\left(B\left(\frac{N}{m+1}+1, \frac{1}{m+1}\right)\right. \\
& \left.-B\left(2^{-m} ; \frac{N}{m+1}+1, \frac{1}{m+1}\right)\right),
\end{aligned}
$$

where $B(a, b) \equiv \int_{0}^{1} t^{a-1}(1-t)^{b-1} \mathrm{~d} t$ (the Euler beta function) and $B(x ; a, b) \equiv \int_{0}^{x} t^{a-1}(1-t)^{b-1} \mathrm{~d} t$ (the incomplete Euler beta function).

$$
\text { 5.2. Case } p=1
$$

For the networks of hierarchy $h=0$

$$
\operatorname{Pr}\left(t_{c}>t\right)=1-\alpha\left(\frac{t}{N}\right)^{m+1}=1-\alpha \rho_{0}^{m+1} .
$$

For the networks of hierarchy $h=1$

$$
\operatorname{Pr}\left(t_{c}>t\right)=1-\rho_{0}+\rho_{0}\left(1-\rho_{1}^{m}\right)\left(1-\alpha \rho_{1}^{m+1}\right)^{m} .
$$

For the networks with a higher degree of hierarchy, $h \geq$ 0 , a recursive formula for the cumulative critical time distribution can be expressed as

$$
\left\{\begin{array}{l}
F_{i}^{(0)}(t)=\alpha \rho_{i}^{m+1} \\
F_{i}^{(h)}(t)=\rho_{i}-\rho_{i}\left(1-\rho_{i+1}^{m}\right)\left(\prod_{d=0}^{h-1}\left(1-F_{i+1}^{(d)}(t)\right)\right)^{m} \\
\operatorname{Pr}^{(h)}\left(t_{c} \leq t\right) \equiv F_{0}^{(h)}(t) .
\end{array}\right.
$$

The mean critical time can be obtained by numerical integration of $\operatorname{Pr}^{(h)}\left(t_{c} \leq t\right)$ :

$$
\left\langle t_{c}\right\rangle=N-\int_{0}^{N} \operatorname{Pr}^{(h)}\left(t_{c} \leq t\right) \mathrm{d} t .
$$

\section{Discussion and conclusions}

\subsection{Number of blocked nodes $Z(t)$}

In all cases the function $Z(t)$, defined as the average number of blocked nodes at time $t$, can be approximated with high accuracy by the power function

$$
Z(t) \propto t^{\beta} .
$$

The $\beta$ exponent depends on the parameters of the network. For $d$-dimensional hypercubic networks (including the 1-dimensional ones, i.e. chains) $\beta=2 d-1$. For modular hierarchical networks $\beta$ depends mainly on the $m$ and $p$ parameters, i.e. on the sizes of the cliques at the lowest hierarchy level, and on the density of the interclique connections. The dependence on the degree of hierarchy $h$ (and on the network size) is weak. It can be explained by the fact that increasing the degree of hierarchy $h$ is a process similar to system rescaling. Thus,

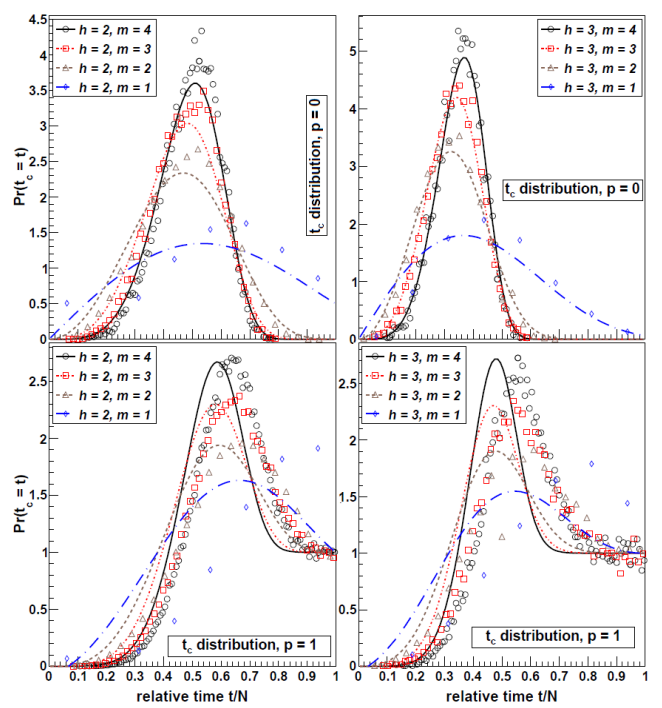

Fig. 4. Critical time distribution for the networks of hierarchy $h=2$ (left) and $h=3$ (right), with $p=0$ (top) and $p=1$ (bottom). Symbols - simulated data, smooth lines - analytical approximations (Eqs. (5.3) and (5.7)).

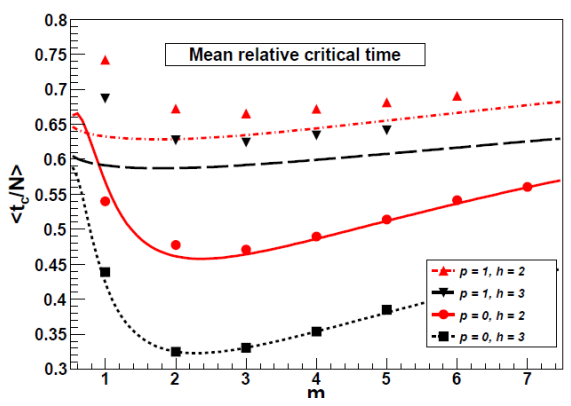

Fig. 5. Mean critical time for various networks. Symbols correspond to the simulated data, lines - to the analytical approximations (Eqs. (5.4) and (5.8)).

one can assume that

$$
\begin{aligned}
& Z^{(h+1)}(\rho) \approx(m+1) Z^{(h)}(\rho)=(m+1) C \rho^{\beta} \\
& \quad=C^{\prime} t^{\beta} .
\end{aligned}
$$

For $p=0$, the $\beta$ parameter can be found analytically: $\beta=m+1$. The results agree with the simulated data. Increasing the density of connections (the $p$ parameter) leads to the increase of $\beta$, up to approximately $m+4$ for $p=1$.

There is an important distinction in the way $Z(t)$ was approximated for hypercubic and hierarchical networks. For hypercubic networks, the number of isolated nodes was calculated using the following approximation: all the blocked nodes were blocked alone, i.e. they do not neighbor with other blocked nodes (of the same community). Although this approximation might seem coarse, the resulting analytical predictions turned out to agree quite well with the simulated data $[11,15]$. For the modular 
hierarchical networks, such an approximation would not be reasonable. As at the lowest level of hierarchy such networks consist of the cliques of $m+1$ nodes, the most probable are the situations when $(m+1) / 2$ nodes are simultaneously blocked.

\subsection{Critical time $t_{c}$}

The second analyzed parameter was the critical time $t_{c}$, i.e. the moment, when the first isolated cluster appears. It is a random variable. The critical time distribution $\operatorname{Pr}\left(t_{c}\right)$ was studied, as well as the mean critical time $\left\langle t_{c}\right\rangle$. More precisely, a critical density (or a critical relative time)

$$
\rho_{c} \equiv \frac{t_{c}}{N}
$$

was often considered instead, so that networks of different sizes could be easily compared.

The $\operatorname{Pr}\left(\rho_{c}\right)$ distribution is always unimodal. The mode (i.e., $\left.\arg \max \operatorname{Pr}\left(\rho_{c}\right)\right)$ decreases with the increase of $h$, and the standard deviation $\sigma\left(\rho_{c}\right)$ decreases with $m$.

For $p=0$, it was possible to find the analytical formulas for both $\operatorname{Pr}\left(t_{c}\right)$ and $\left\langle t_{c}\right\rangle$. The distribution $\operatorname{Pr}\left(t_{c}\right)$ is a polynomial of degree $m(m+1)\left((m+1)^{h}-1\right)$ (Eq. $(5.3))$, and the average $\left\langle t_{c}\right\rangle$ is a scaled difference of two Euler beta functions (Eq. (5.4)). The average critical density $\left\langle\rho_{c}\right\rangle$ decreases with $h$ and for a fixed $h$ it reaches the minimum for $m \approx 2$ (Fig. 5 ).

For $p=1$, the distribution $\operatorname{Pr}\left(\rho_{c}\right)$ reaches a constant, non-zero value for $\rho_{c} \in[1-\epsilon, 1]$ (e.g., for $h \leq 3, \epsilon \approx 0.1$ ), which means that processes when the blocked clusters first appear at the very end of the evolution are not unlikely.

The values of $\left\langle\rho_{c}\right\rangle$ can be compared with the ones obtained for hypercubic networks. Similar trends can be observed in hypercubic and hierarchical networks: $\left\langle\rho_{c}\right\rangle$ decreases with the network size $N$ and increases with the average degree. However, for the modular hierarchical networks the dependence of $\left\langle\rho_{c}\right\rangle$ on the average degree (which equals $m$ for $p=0$ and rises with $p$ ) is very weak compared to hypercubic networks. Typical values of $\left\langle\rho_{c}\right\rangle$ for hierarchical networks correspond to the ones obtained for two- or three-dimensional networks, even for $m \gg 3$.

\section{Acknowledgments}

The work was supported by FP7 FET Open project Dynamically Changing Complex Networks - DynaNets EU Grant Agreement Number 233847, European COST Action MP0801 Physics of Competition and Conflicts and Polish Ministry of Science Grant 1029/7.PR UE/2009/7 and Grant 578/N-COST/2009/0.

\section{References}

[1] E. Ravasz, A.-L. Barabási, Phys. Rev. E 67, 026112 (2003).

[2] S. Komosa, J.A. Hołyst, Ising model at hierarchical network, to be published.

[3] M.F. Laguna, S. Risau Gusman, G. Abramson, S. Gonçalves, J.R. Iglesias, Physica A 351, 580 (2005).

[4] S. Galam, A. Vignes, Physica A 351, 605 (2005).

[5] K. Suchecki, J.A. Hołyst, Acta Phys. Pol. B 36, 2499 (2005).

[6] I. Dornic, H. Chat, J. Chave, H. Hinrichsen, Phys. Rev. Lett. 87, 045701 (2001).

[7] G. Deffuant, F. Amblard, G. Weisbuch, T. Faure JASSS 5 (2002).

[8] S. Galam, Eur. Phys. J. B 25, 403 (2002).

[9] S. Lee, P. Holme, Z.-X. Wu, Phys. Rev. Lett. 106, 028702 (2011).

[10] C. Castellano, S. Fortunato, V. Loreto, Rev. Mod. Phys. 81, 591 (2009).

[11] J. Sienkiewicz, J.A. Hołyst, Phys. Rev. E 80, 036103 (2009).

[12] P. Sjögren, Biological Journal of the Linnean society 42, 135 (1991).

[13] T.C. Schelling, J. of Math. Soc. 1, 143 (1971).

[14] D. Stauffer, S. Solomon, Eur. Phys. J. B 57, 473 (2007).

[15] J. Sienkiewicz, G. Siudem, J.A. Hołyst, Phys. Rev. E 82, 057101 (2010). 